\title{
New insight to the characteristics and clinical course of clusters of patients with imaging confirmed disc-related sciatica
}

\section{Running title}

Clusters and clinical course of disc-related sciatica

Author details

S Stynes ${ }^{1,2 *}$, L Grøvle ${ }^{3}$, AJ Haugen ${ }^{3}$, K Konstantinou $^{1,2}$, M Grotle $^{4,5}$

Siobhán Stynes

${ }^{1}$ Primary Care Centre Versus Arthritis, Research Institute for Primary Care and Health Sciences, Keele University, Keele, Staffordshire, ST5 5BG, UK

${ }^{2}$ Haywood Hospital, Midlands Partnership NHS Foundation Trust, Stoke-on-Trent, Staffordshire, ST6 7AG, UK.

Lars Grøvle

${ }^{3}$ Department of Rheumatology, Østfold Hospital Trust, PB 300, 1714 Grålum, Norway

Anne Julsrud Haugen

${ }^{3}$ Department of Rheumatology, Østfold Hospital Trust, PB 300, 1714 Grålum, Norway

Kika Konstantinou

${ }^{1}$ Primary Care Centre Versus Arthritis, Research Institute for Primary Care and Health Sciences, Keele University, Keele, Staffordshire, ST5 5BG, UK

${ }^{2}$ Haywood Hospital, Midlands Partnership NHS Foundation Trust, Stoke-on-Trent, Staffordshire, ST6 7AG, UK.

Margreth Grotle

${ }^{4}$ Faculty of Health Science, OsloMet - Oslo Metropolitan University, Oslo, Norway

${ }^{5}$ Research and Communication Unit for Musculoskeletal Health, Oslo University Hospital, Oslo, Norway

* Corresponding author

Dr Siobhán Stynes, Arthritis Research UK Primary Care Centre, Research Institute for Primary Care and Health Sciences, Keele University, Keele, Staffordshire, ST5 5BG, UK

Tel: 00441782733925

Fax: 00441782734719

Email: s.stynes@keele.ac.uk

\section{Category}

Original article

This article has been accepted for publication and undergone full peer review but has not been through the copyediting, typesetting, pagination and proofreading process, which may lead to differences between this version and the Version of Record. Please cite this article as doi: 10.1002/ejp.1475

This article is protected by copyright. All rights reserved. 


\title{
Funding source
}

This research was supported by National Institute for Health Research (NIHR) funding awards to Siobhan Stynes NIHR/CNO Clinical Doctoral Research Fellowship (CDRF-2010-055) and Kika Konstantinou HEFCE/NIHR Senior Clinical Lectureship. Lars Grøvle and Anne Julsrud Haugen were supported by a research grant from the Eastern Norway Regional Health Authority (2007049). The views expressed in this paper are those of the authors and not necessarily those of the NHS, the NIHR, or the Department of Health and Social Care.

\section{Conflicts of interest}

No conflicts of interest

\section{Significance}

Using data from a large observational prospective study, this work identifies four distinct clusters of patients with imaging confirmed disc-related sciatica. This classification could be used when considering prognosis and management with sciatica patients at their initial consultation.

\begin{abstract}
Background

Referral to secondary care is common for a considerable proportion of patients with persistent sciatica symptoms. It is unclear if information from clinical assessment can further identify distinct subgroups of disc-related sciatica, with perhaps different clinical courses.

Aims

This study aims to identify and describe clusters of imaging confirmed disc-related sciatica patients using latent class analysis, and compare their clinical course.
\end{abstract}

\section{Methods}

The study population were 466 patients with disc-related sciatica. Variables from clinical assessment were included in the analysis. Characteristics of the identified clusters were described and their clinical course over two years, was compared.

\section{Results}

A four cluster solution was optimal. Cluster $1(n=110)$ had mild back and leg pain; cluster $2(n=59)$ had moderate back and leg pain, cluster $3(n=158)$ had mild back pain and severe leg pain; cluster 4 $(n=139)$ had severe back and leg pain. Patients in cluster 4 had the most severe profile in terms of disability, distress and comorbidity and the lowest reported global change and the smallest proportion of patients with a successful outcome at two years. Of the 135 patients who underwent surgery, $42 \%$ and $41 \%$ were in clusters 3 and 4 respectively.

\section{Conclusions}

Using a strict diagnosis of sciatica, this work identified four clusters of patients primarily differentiated by back and leg pain severity. Patients with severe back and leg pain had the most severe profile at baseline and follow-up irrespective of intervention. This simple classification system may be useful when considering prognosis and management with sciatica patients.

This article is protected by copyright. All rights reserved. 


\section{Introduction}

For most patients with sciatica (lumbar nerve root pain), the clinical course is generally favourable. However, a considerable proportion, up to $30 \%$ (Koes et al., 2007), usually those with more severe initial symptoms, will continue to suffer from pain and related disability after one year. In most cases, sciatica is caused by lumbar disc herniation and is associated with pain in the leg, usually below the knee with or without accompanying numbness, reflex change or muscle weakness (Koes et al., 2007). Referral to secondary care is common among the group of patients with persistent severe symptoms, for further diagnostic tests, injections for pain relief or spinal surgery. This contributes to the considerable costly impact of sciatica to society, with an estimated impact to the UK economy of $£ 268$ million direct costs and $£ 1.9$ billion indirect costs per annum (Foster et al., 2017).

Currently sciatica management is mainly a stepped care approach, starting with non-invasive treatment and progressing to more invasive options if symptoms persist and if appropriate. The effectiveness of medications in managing sciatica in primary care, is unclear (Pinto et al., 2012), active physiotherapy treatment is beneficial for some patients but evidence to support its efficacy is not strong (Albert and Manniche, 2012; Luijsterburg et al., 2008), and both spinal injections and surgery show faster improvement in the shorter term but longer term outcomes are similar irrespective of treatment (Chou et al., 2015; Peul et al., 2007; Valat et al., 2010). It is clear that some patients with sciatica respond more favourably to certain interventions (Lurie et al., 2014), but we do not know how to match patients to treatments, more effectively.

When comparing outcomes among an overall group of 'sciatica' patients, the differences are diluted. Identification of subgroups of patients with disc-related sciatica may help clinicians to tailor treatment according to patient characteristics and give more informed guidance on likely outcomes. Previous work derived from a primary care cohort of patients which included a wide spectrum of back-related leg pain, in terms of symptoms severity, identified five hitherto unrecognised clusters of low back-related leg pain (LBLP) patients, including sciatica (nerve root/radicular pain), with different characteristics and clinical course, using clinical assessment information (Stynes et al., 2018). Three clusters identified were considered to represent patients with mild, moderate or severe sciatica symptoms, a cluster with leg pain but not due to nerve root involvement (referred pain) and a cluster that was clinically difficult to define, labelled "atypical sciatica".

The work presented in this paper replicates the statistical technique of cluster identification previously used in LBLP patients, but with a much tighter definition of sciatica that minimises diagnostic uncertainty. It also tests if clusters already identified in a primary care population are similar to those seen in sciatica patients recruited from a secondary care setting. The aim of this work was to identify clusters of patients with imaging (magnetic resonance imaging (MRI) or computer tomography (CT)) confirmed disc-related sciatica, using items from baseline clinical assessment.

\section{Methods}

The study used data from patients with sciatica and lumbar disc herniation confirmed by imaging, who were participating in a prospective, multicentre, observational study. First, the optimal number of clusters of patients with disc-related sciatica were identified using latent class analysis, second, the clusters were compared on baseline sociodemographic, disability, health, psychosocial and workrelated characteristics, third, the clinical course of the clusters in terms of pain and disability over 2

This article is protected by copyright. All rights reserved. 
years was determined, and fourth, the influence of management strategies on improvement of patients in the various clusters, was described.

Details of the cohort and longitudinal data results have been published (Grovle et al., 2008; Haugen et al., 2012). A brief overview of the study methods and baseline cohort characteristics are given here.

Patients were recruited from specialty back pain clinics at four hospitals in southeast Norway. Referrals to the clinics were from primary health care settings. At the routine back clinic appointment, eligible patients were informed about the study by a physician or physiotherapist. Inclusion criteria were age over 18 years and radiating pain below the knee or paresis, and a disc herniation at the corresponding level and side according to MRI or computed tomography (CT). Exclusion criteria were prior surgery at the same disc level, fracture, infection, malignancy, pregnancy, and lack of fluency in Norwegian. At the first visit, all eligible and consenting participants underwent a standardised clinical assessment by a physiotherapist or physician and completed a baseline self-report questionnaire. Follow-up postal questionnaires were sent to participants at 3, 6, 12 and 24 months. At the baseline clinical assessment, the clinician gave patients usual information about their sciatica condition, general advice to stay active and use pain medication as needed. In patients with severe symptoms, surgery was performed the discretion of the individual surgeon (Grøvle et al., 2014).

At baseline, data were available for 466 patients (female 198 (43\%), mean age 43.6 (sd 11.5)). The 3, 6, 12 and 24-month questionnaires were answered by 434 (93.1\%), 423 (90.8\%), 409 (87.8\%) and $380(81.5 \%)$ patients, respectively.

A total of $41 \%$ ( $n=192)$ of patients had leg pain duration of less than three months, $43 \%$ ( $n=156)$ had leg pain duration of 4-6 months and $25 \%(n=116)$ had leg pain for longer than 6 months. Average pain intensity during the previous week (0-100) was 42.6 (sd 30.0) for back pain and 63.2 (sd 28.2) for leg pain. Mean disability assessed by the Maine Seattle Back Questionnaire (MSBQ; 0-12) was 8.1 (sd 2.6). One fifth of the sample ( $n=92)$ were currently in work, $11 \%$ were on partly sick leave $(n=51)$ and $57 \%$ on sick leave/disability pension $(n=267)$.

Neurological examination findings showed 203 patients (45.6\%) had a myotome deficit, 213 (45.7\%) a reflex deficit and $273(58.6 \%)$ patients had a sensory deficit, with $81 \%(n=375)$ of patients overall having a neurological deficit in the lower limb. A positive SLR was recorded in $57 \%(n=267)$ of patients. Further details of the physical examination measurements are given in Table 1.

During the 2-year follow-up, $29 \%$ ( $n=135)$ of patients were treated surgically for their disc-related sciatica, 23 of whom (17\%) were lost to follow-up at 2 years. Most patients (81\%) underwent surgery within 3 months of the baseline assessment.

\section{Variables included in the latent class modelling}

Eight variables were chosen a priori from the set of available self-report and clinical assessment variables. These variables were chosen to mirror as much as possible, variables used in previous latent class (LC)/cluster identification in a cohort of LBLP patients (Stynes et al., 2018), and reflect criteria that best distinguish clinical features of sciatica (Lin et al., 2014; Stynes et al., 2016).

Two of the included variables were on a continuous scale (leg pain intensity 0-10; back pain intensity $0-10)$. The remaining variables were binary (yes/no): sensory bothersome; leg pain worse than back pain; positive straight leg raise, myotomal (strength) deficit, reflex deficit, sensory deficit. (See Table 1 for description of variables).

This article is protected by copyright. All rights reserved. 


\section{Table 1 Description of variables used for latent class modelling}

\section{Latent class model development}

The aim of LC modelling is to identify meaningful groups or clusters, previously unobserved in a population, that are similar in their responses to measured variables (Muthen, 2004). The clusters are fitted consecutively starting with a two cluster solution. The optimal number of clusters are identified based on the following criteria:

(i) Bayesian Information Criterion (BIC) (a lower BIC is preferred) and the bootstrapped parametric likelihood ratio test (LRT) which assesses if the addition of a cluster significantly improves the model fit (Nylund et al., 2007); entropy measuring the overlap between clusters ( 0 to 1 , closer to 1 is optimal) (Collins and Lanza, 2010) and average posterior probabilities to indicate clear distinction for individuals allocated to that cluster (Clark et al., 2006) (values should be greater than 0.7).

(ii) A cluster size of at least $10 \%$ of the sample (Green et al 2015, Nasserinejad et al 2017).

(iii) Conditional mean scores (continuous variables) and item conditional probabilities (range 0 to 1 ) to the binary variables in the LC modelling. A probability of 1 means that all patients in that cluster responded "yes" to that item e.g. all had 'a sensory deficit". Probabilities closer to 0.5 reflect more uncertainty in distinguishing clusters (Green et al., 2015).

To help assign a descriptive label to the identified clusters, the clinical meaningfulness and interpretability of the clusters were considered.

\section{Cluster characteristics}

The identified clusters were described and compared across baseline characteristics which included: age, gender, smoking and work status, history of previous sciatica and duration of symptoms.

Emotional distress was measured using the Hopkins Symptom Check List (HSCL- 25) (Derogatis et al., 1974) which includes 25 items to assess anxiety and depression symptoms during the previous week. Each item has four response categories ranging from not at all (1) to extremely (4) and the score is calculated as the sum of all items divided by the number of items answered. A clinical cut-off of 1.75 is commonly used to define symptomatic cases (Sandanger et al., 1998). The Sciatica Bothersomeness Index (SBI) (0-24), based on a composite score of self-reported ratings (0-6) of bothersomeness of (i) leg pain, (ii) sensory disturbance in the lower limb (iii) leg/foot weakness (iv) back or leg pain while sitting (Patrick et al., 1995). Higher scores indicate worse symptoms. The bodily pain and physical functioning subscales of the quality of life SF-36 questionnaire (Ware, 2000) were used. Scores range from 0 to 100 , higher values indicate better health.

The comorbid subjective health complaints (SHC) (0-27) inventory included 27 health complaints such as muscular pain, stomach pain and discomfort, hot flushes, extra heart beats, sleep problems, tiredness, dizziness (Eriksen et al.,1999; Ihlebaek et al., 2007). Patients graded the intensity of each complaint during the previous month as not at all (0), a little (1), some (2) or severe (3). Each item was dichotomised to absent $(0)$ or present $(1,2,3)$ (Haugen et al., 2011). The Tampa Scale for Kinesiophobia (TSK) [13-52], a 13-item, four-point questionnaire where higher scores indicate increased kinesiophobia (fear of movement) (Haugen et al., 2008; Vlaeyen et al., 1995).

Patients who underwent surgery for disc herniation reported the date of surgery in follow-up questionnaires.

\section{Clinical course}

The clinical course of the identified clusters was examined over a 24-month period, for leg pain, back pain, and back and leg pain related disability. Leg and back pain intensity during the previous week were measured using a 100-mm Visual Analogue Scales (VAS) with $0=$ No pain and $100=$ Maximum

This article is protected by copyright. All rights reserved. 
pain at the scale endpoints. Disability was measured using the Maine-Seattle Back Questionnaire (MSBQ), a 12 item scale with scores ranging from 0 to 12 with higher scores indicating greater disability (Atlas et al., 2003; Grovle et al., 2008).

At each follow-up time point, participants rated the change of their sciatica/back problem from baseline on a global perceived change (GPC) scale as either completely gone, much better, better, a little better, no change, a little worse, and much worse The proportion of patients that proceeded to have spinal surgery was described for each cluster, and for those patients who did and did not have surgery, the proportion with a successful outcome (dichotomised as a MBSQ < 5 score) was described as well as the proportion who self-reported recovery of their sciatica/back problem ('completely recovered' or 'much better' on the GPC). $M S B Q \geq 5$ is a validated outcome measure in this sciatica cohort (Haugen et al., 2011) with a cut off of 4.5 having the highest sensitivity and specificity against the GPC score as an external criterion.

\section{Analyses}

Each characteristic was compared across the number of identified clusters using ANOVA for continuous variables (Kruskall Wallis test when normality and homogeneity of variance assumptions were not met) and Pearson's Chi squared test (Fisher's exact test used for cell frequencies $<5$ ) for categorical variables. Analyses were two tailed and considered statistically significant if $p<0.05$. LC modelling was performed in Mplus version 7 (Muthen and Muthen Los Angeles, CA).

Ethical approval for the cohort study was approved by the Regional Committee for Medical Research Ethics (East-Norway) and The Ombudsmann for Privacy in Research at the Norwegian Social Science Data Services.

\section{Results}

Latent class modelling was performed on the baseline data of 466 patients.

\section{Model development}

A four cluster LC solution was optimal when statistical indices of fit (table 2) and clinical meaningfulness of the clusters was considered. The bootstrapped LRT $p$-value remained significant for all cluster solutions suggesting the model fit improved every time a cluster was added to the model. There was high probability of individuals in the 4 cluster solution being classified to their allocated group, with all average probabilities $>0.92$. Compared to the 2 and 3 cluster solution, the entropy was highest $(0.875)$ and BIC was lowest. In the 5 cluster solution, entropy was the same value as the 4 cluster solution and BIC was lower, however when the clusters were compared on clinical meaningfulness, the item response probabilities of categorical variables of two of the clusters was identical and there were only small differences in baseline mean leg and back pain intensity values between these two clusters. The 6 cluster solution had a small sample size of less than $10 \%$ in one cluster ( $n=35$ ) and on examination of the additional two clusters, they were similar to the mild and severe cluster with slightly different pain intensity levels. Therefore, it was decided that including an additional one or two clusters, added to the complexity of the classification without offering further clinical value.

This article is protected by copyright. All rights reserved. 
Table 2 Statistical indices of fit of the latent cluster models of disc-related sciatica patients Description of clusters

The 4 cluster response probabilities to the clinical assessment items and their corresponding back and leg pain intensity (converted from 0-100 to a 0-10 scale) are displayed in Figure 1 . The most discriminating items for the four clusters were pain intensity and the relationship between back and leg pain intensity (see Table 3). Pain intensity is described as mild $(\leq 3 / 10)$, moderate $(4$ to $7 / 10)$ and severe $(>7 / 10)$ intensity). Clusters are labelled according to leg and back pain intensity, sorting them from minimum to maximum leg and back pain. Patients in cluster $1(n=110)$ had low intensity back and leg pain with leg pain slightly higher than back pain intensity. We labelled this cluster 'mild'. Patients in cluster $2(n=59)$ had moderate intensity back and leg pain with back pain slightly higher than leg pain intensity labelled as 'moderate'. Patients in cluster $3(n=158)$ had low intensity back pain and high intensity leg pain labelled as 'mild back and severe leg'. Patients in cluster 4 ( $n=139)$ had high intensity back and leg pain with leg pain greater than back pain labelled as 'severe'. Probability of being bothered by paraesthesia in the legs (sensory bothersome) was least for patients in clusters 1 (0.24) and 2 (0.34). Information from the neurological assessment of myotome or reflex deficit did very little to improve the discrimination between the clusters, with probabilities ranging from 0.37 (cluster 2, myotome deficit) to 0.54 (cluster 3, reflex deficit) across the clusters. Sensory deficit was slightly more discriminatory, with lowest probability in cluster $2(0.44)$ to highest in cluster 3 (0.69). Probability of a positive SLR was slightly more discriminatory with patients in cluster 1 having the lowest probability of a positive SLR (0.35), and the highest probability in clusters 3 (0.70) and 4 (0.67).

Fig. 1 Four cluster latent class analysis solution. Item response probabilities of categorical variables (left vertical axis) and baseline mean leg and back pain intensity (right vertical axis) Cluster characteristics

The clusters differed significantly in all baseline characteristics with the exception of age, gender, symptoms duration and level of imaging confirmed disc herniation (Table 3). Observed differences across all other measures were explained by the mild cluster which scored "low" on most variables compared to the other three clusters. Clusters 2 (moderate) and 3 (mild back, severe leg) had similar profile scores across all domains. The greatest proportion of smokers was in cluster 4 (severe) (52\%), the lowest in cluster 2 (moderate) (34\%). Patients in cluster 4 (severe) had the most severe profile including the highest levels of disability (MSBQ), distress (HSC) comorbidity (SHC), fear avoidance (FABQ), sciatica bothersomeness (SBI), kinesiophobia (TSK) and poorest general health score (PF36).

\section{Table 3 Baseline characteristics of the total sample and 4 clusters with disc-related sciatica Clinical Course}

The response rate for individual clusters to the questionnaires were almost identical at 3, 6 and 12 months to the overall average response rates at each time point. At 2 years, the response rates from clusters 2 (moderate) and 4 (severe) were slightly lower than the overall average with responses from $78 \%$ and $77 \%$ of patients in clusters 2 and 4 respectively.

Leg pain and disability scores improved over the two-year period for all clusters (figure 2), with the greatest improvement seen from baseline to 3 months after which the improvement was more gradual. The same trend was seen in back pain for clusters 2 (moderate) and 4 (severe), whereas back pain scores for clusters 1 (mild) and 3 (mild back, severe leg) remained consistently low over the two years.

This article is protected by copyright. All rights reserved. 
The lowest reported global perceived change (recovery/improvement) (sciatica/back pain completely gone/much better) was reported by patients in cluster 4 (severe) at one and two years (41\% of patients at 12 months, $44 \%$ at 24 months) followed by cluster 2 (moderate) ( $47 \%$ of patients at 1 year, 57\% at 2 years). At 12 months, clusters 1 (mild) and 3 (mild back, severe leg) reported $60 \%$ and $64 \%$ recovery respectively, increasing to $66 \%$ for both clusters at 24 months.

\section{Fig. 2 Clinical course over 24 months of back pain (top figure), leg pain (middle figure) and disability (bottom figure) for the 4 clusters \\ Outcomes in surgical patients}

Of the 135 patients who underwent surgery, $42 \%(n=56)$ and $41 \%(n=55)$ were in clusters 3 (mild back, severe leg) and 4 (severe), respectively, $7 \%$ were in cluster 1 (mild) $(n=10)$ and $10 \%$ in cluster 2 (moderate) ( $\mathrm{n}=14)$. At 12 months, the proportion of patients with a successful outcome (MSBQ $<5)$ following surgery ranged from $78 \%$ (cluster 2 ) to $52 \%$ (cluster 4 ). At 24 months, cluster 4 had the lowest proportion of patients with a successful outcome (48\%) following surgery. The proportion with a successful outcome was the same amongst those who did and did not have surgery.

\section{Discussion}

This study used the statistical method of latent class modelling to reveal four distinct clusters of patients with disc-related sciatica. The clusters give further insight into the variability in the characteristics and clinical course of patients within the clear diagnostic classification of imaging confirmed disc-related sciatica, not previously identified in the literature. The clusters are modelled based on their back and leg pain intensity and response to items from clinical assessment. The four identified clusters of sciatica patients are primarily differentiated by the intensity of their back and leg pain and the relationship between back and leg pain intensity. Leg pain intensity was greater than back pain in all clusters except cluster 2 (moderate). Patients with severe back and leg pain (cluster 4 ) had the most severe profile at baseline, scoring high on measures covering different domains in the biopsychosocial model (physical, psychological and social domains). Cluster 4 also had the least favourable pain and disability outcomes at 2 years and the lowest proportion of people in the cluster reporting improvement.

The presence of any neurological deficit is well documented and accepted as a diagnostic criterion for sciatica (Lin et al.,2014; Stynes et al., 2016). However in this analysis, items from the neurological examination did little to differentiate between the clusters because the number of patients with myotome, reflex or a sensory neurological deficit were relatively evenly spread across the four clusters. It was not considered necessary to model clusters using individual nerve root level myotome, reflex or sensory deficits as there is no evidence in the literature to suggest that this level of detail is implicated in the impact or prognosis of sciatica. Furthermore, the distribution of the level of disc herniation confirmed by imaging was evenly spread among the four clusters. Patients in the two clusters with very high leg pain intensity (clusters 3 (mild back, severe leg) and 4 (severe)) had more sensory bothersomeness and positive SLR than clusters 1 (mild) and 2 (moderate). In this sciatica cohort, patients' perception of sensory bothersomeness was associated with greater impact on work function and emotional distress (Grøvle et al., 2013). Although most of the cohort were in employment, $80 \%$ were not at work due to sick leave or disability pension at baseline. This figure is very high but similar proportions of sciatica patients who took time off work (79\%) were seen in a Dutch randomised clinical trial comparing surgery with conservative treatment (Peul et al., 2007). In this Norwegian secondary care cohort, pain and disability levels are also similar to other secondary care sciatica cohorts (Peul et al., 2007).

This article is protected by copyright. All rights reserved. 
Proportions of patients with "chronic" symptoms, i.e. more than 3 months, were similar across the 4 clusters, ranging from $60 \%$ (cluster 3 , mild back, severe leg) to $69 \%$ (cluster 2 , moderate). The highest proportion of smokers was seen in cluster 4 (severe), and comorbid subjective health complaints (SHC) were highest in clusters 2 and 4. Previous analysis on this cohort showed that smoking, alongside male gender, higher scores for back pain and not having surgery, were associated with poor outcome at 12 months and a high score for comorbid SHC was the only variable that predicted a poor outcome / non-success at 12 and 24 months (Haugen et al., 2012).

The clinical course for leg pain and disability was favourable for all 4 clusters, with most improvement seen in the first 3 months. This early improvement is confirmed in other sciatica trajectory studies (Stynes et al., 2018) and is also seen in cohorts with non-specific low back pain (Axen \& Leboeuf-Yde, 2013; Downie et al., 2016). An interesting observation is the early improvement in pain and disability across the 4 clusters despite approximately $2 / 3$ of patients in all clusters having their pain for over 3 months. Although this phenomenon of early improvement is difficult to explain in an observational cohort, the process of referral to a specialist, inclusion to a study, and subsequent information and advice about their diagnosis and treatment received may have contributed to initial symptom improvement. In those that received surgery, the majority had their surgery within 3 months of inclusion to the study. Patients in the four clusters remained in some pain and disability at 12 months, but disability scores for cluster 4 (severe) remained at least 5 on the MSBQ at 12 and 24 months which is considered a poor outcome. When patients were asked to rate their own recovery, the proportion reporting they were completely better or much better, reflected the trends seen in disability scores, with the lowest proportion seen in cluster 4 (severe).

\section{Strengths and Limitations}

The strengths of this work include using a rich data set to combine a statistical technique to identify clusters of patients, with clinical judgement to assist interpretation of the clusters. The description of the clusters is based on a comprehensive dataset of self-report and clinical assessment information. The sample population represents a group with a clear sciatica definition based on clinical diagnosis with concordant MRI or CT imaging findings. With the exception of surgical cohorts, sciatica definition is inconsistent in the literature (Lin et al., 2014), making it a challenge to compare characteristics and outcomes across sciatica studies. Mapping the clinical course of the 4 clusters is robust as there was a high response rate $(>80 \%)$ at each time point.

A limitation of the work is that the identified clusters are based on the chosen variables to model the groups and arguably more distinct or clinically relevant clusters could have been identified with the inclusion of different variables. Variable selection was decided a priori and chosen to reflect baseline information gained from a clinical assessment, in order to explore if this information could help differentiate or distinguish hidden or latent groups not readily discerned by the clinician.

A number of patients eligible for inclusion in the cohort study were either not invited or declined participation. There is limited data available on these patients therefore participation bias is possible if those who did not participate differed from participants on key characteristics.

To explore generalisability of these clusters, they should be confirmed and validated in other discrelated secondary care sciatica cohorts.

This article is protected by copyright. All rights reserved. 


\section{Clinical Implications}

For patients with MRI confirmed disc-related sciatica, this work reveals the variability of presentation and clinical course and identifies more homogenous groups.

The different trajectories over time of the individual clusters, gives more information as opposed to examining the average clinical course of the whole cohort. This detail could be helpful to patients when discussing their potential prognosis, and to clinicians when considering available management options.

Of the 135 patients that underwent surgery, the majority were in the two clusters with severe profiles of pain intensity, comorbidities, disability and distress. We are unable to infer from our data if this patient profile is more likely to have a higher influence on either the patient's or surgeon's decision for surgical treatment, and which aspect of the patient's presentation had a higher influence on the decision. Published work has demonstrated that for patients, the decision whether or not to undergo spinal surgery is a complex, multi-factorial process (Lam and Loke 2017) and a survey of spinal surgeons showed that the surgeons own experience and 'gut feeling' was the highest influence in the decision making process (Sollman et al 2018). This cohort study did not include qualitative methods to explore the clinical decision making process for undergoing surgery, but is undoubtedly an area that would benefit from further empirical evidence.

The observed differences in clinical course and outcomes of the severe leg pain sciatica patients, with severe back pain (cluster 4 ) and mild back pain (cluster 3 ), might reflect the different pain mechanisms involved. Irrespective of the clinical management (conservative and/or surgical), those with severe back pain did less well, reflecting current management strategies inadequacy in relieving back pain in sciatica.

An individual's experience of low back pain with or without sciatica may be due to an interplay of nociceptive, inflammatory, neuropathic and central sensitization pain perception modified by factors including genotype and psychosocial characteristics (Vardeh et al 2016).

Even in the case of MRI confirmed disc-related sciatica, effectiveness of surgery to remove the compressive mechanism or epidural steroid injection to target the inflammatory component, has varied outcomes (Vardeh et al 2016, Jacobs et al., 2011).

Whether this is because the neuropathic component of low back pain is not addressed, it is difficult to ascertain, but it may be a possibility, as the initial nocioceptive activity from a degenerative intervertebral disc may progress to involve microscopic nerve fibres causing local neuropathic pain (Baron et al 2016, Vardeh et al 2016). Neuropathic pain is a challenge to manage, and many patients with persistent LBP might have neuropathic pain that does not respond to existing treatments (Baron et al 2016). Previous longitudinal modelling on a cohort of patients with low back-related leg pain identified a "persistent severe" and "improving severe" group. The characteristics that distinguished the persistent severe group were the patients were more likely to report leg pain of potential neuropathic nature (measured using the self-report Leeds Assessment Neuropathic Symptoms and Signs (S-LANSS) questionnaire) than those in the "improving severe" group. In our sciatica cohort, features of neuropathic pain were not measured by self-report questionnaire or concomitant clinical examination (e.g. Quantitative Sensory Testing (QST)) hence the potential role of neuropathic pain within the clusters remains speculative.

Literature shows a limited number of factors associated with outcome in sciatica, all indicative of condition severity or perceived severity (Ashworth et al., 2011; Fjeld et al., 2017; Konstantinou et al., 2018; Verwoerd et al., 2013, Grovle et al., 2013). Currently for patients with disc-related sciatica,

This article is protected by copyright. All rights reserved. 
there is no consensus on which treatment works best for whom or the optimal timing of interventions. This work uses diagnostic information and clinical course observations in patients with a diagnosis of disc-related sciatica, which could be used to illustrate their potential clinical course based on baseline presentation and plan management accordingly, within the limitations of current effective management options for this condition.

\section{Conclusion}

The four clusters of sciatica patients identified in this work are primarily differentiated by the severity of back and leg pain and the clinical course of those with more severe back pain is less favourable. Patients with high intensity back and leg pain had the most severe profile at baseline and follow-up irrespective of intervention. This simple classification system may be useful when considering prognosis and treatment options with sciatica patients at their initial consultation and encourage clinicians and researchers to also focus on addressing back pain levels in patients with sciatica.

\section{Author contributions}

LG, AJH and MG participated in the design, set up and data collection for the original cohort study. All authors participated in developing the study design and analysis plan. SS completed the analysis and was in charge of writing the manuscript and coordinated publication activities. All authors discussed the results and commented on the manuscript.

\section{References}

Albert, H. B., \& Manniche, C. (2012). The efficacy of systematic active conservative treatment for patients with severe sciatica: a single-blind, randomized, clinical, controlled trial. Spine (Phila Pa 1976), 37(7), 531-542. doi: 10.1097/BRS.0b013e31821ace7f .

Ashworth, J., Konstantinou, K., \& Dunn, K. M. (2011). Prognostic factors in non-surgically treated sciatica: A systematic review. BMC Musculoskelet Disord, 12, 208. doi: 10.1186/1471-247412-208.

Atlas, S. J., Deyo, R. A., van den Ancker, M., Singer, D. E., Keller, R. B., \& Patrick, D. L. (2003). The Maine-Seattle back questionnaire: a 12 -item disability questionnaire for evaluating patients with lumbar sciatica or stenosis: results of a derivation and validation cohort analysis. Spine (Phila Pa 1976), 28(16), 1869-1876. doi: 10.1097/01.brs.0000083205.82614.01.

Axen, I., \& Leboeuf-Yde, C. (2013). Trajectories of low back pain. Best Pract Res Clin Rheumatol, 27. doi: 10.1016/j.berh.2013.10.004.

Baron, R., Binder, A., Attal, N., Casale, R., Dickenson, A.H., Treede, R.D. (2016). Neuropathic low back pain in clinical practice. Eur J Pain, 20(6), 861-873.

Chou, R., Hashimoto, R., Friedly, J., Fu, R., Bougatsos, C., Dana, T., . . Jarvik, J. (2015). Epidural corticosteroid injections for radiculopathy and spinal stenosis: A systematic review and meta-analysis. Ann Intern Med, 163(5), 373-381. doi: 10.7326/M15-0934.

Clark, D. B., Jones, B. L., Wood, D. S., \& Cornelius, J. R. (2006). Substance use disorder trajectory classes: diachronic integration of onset age, severity, and course. Addictive Behaviors, 31(6), 995. doi: S0306-4603(06)00086-4.

Collins, L., \& Lanza, S. (2010). Latent class and latent transition analysis: With applications in the social, behavioural, and health sciences. New York John Wiley \& Sons.

Costa Lda, C., Koes, B. W., Pransky, G., Borkan, J., Maher, C. G., \& Smeets, R. J. (2013). Primary care research priorities in low back pain: an update. Spine (Phila Pa 1976), 38(2), 148-156. doi: 10.1097/BRS.0b013e318267a92f.

Derogatis, L. R., Lipman, R. S., Rickels, K., Uhlenhuth, E. H., \& Covi, L. (1974). The Hopkins Symptom Checklist (HSCL): a self-report symptom inventory. Behav Sci, 19(1), 1-15.

This article is protected by copyright. All rights reserved. 
Downie, A. S., Hancock, M. J., Rzewuska, M., Williams, C. M., Lin, C. W., \& Maher, C. G. (2016). Trajectories of acute low back pain: a latent class growth analysis. Pain, 157(1), 225-234. doi: 10.1097/j.pain.0000000000000351.

Eriksen, H. R., Ihlebaek, C., \& Ursin, H. (1999). A scoring system for subjective health complaints (SHC). Scand J Public Health, 27(1), 63-72.

Fjeld, O., Grotle, M., Siewers, V., Pedersen, L. M., Nilsen, K. B., \& Zwart, J. A. (2017). Prognostic Factors for Persistent Leg-Pain in Patients Hospitalized With Acute Sciatica. Spine (Phila Pa 1976), 42(5), E272-e279. doi: 10.1097/brs.0000000000001773.

Foster, N. E., Anema, J. R., Cherkin, D., Chou, R., Cohen, S. P., Gross, D. P., . . Woolf, A. (2017). Prevention and treatment of low back pain: evidence, challenges, and promising directions. The Lancet. doi: 10.1016/S0140-6736(18)30489-6.

Foster, N. E., Konstantinou, K., Lewis, M., Ogollah, R., Dunn, K. M., van der Windt, D., ... Hay, E. M. (2017). The clinical and cost-effectiveness of stratified care for patients with sciatica: the SCOPiC randomised controlled trial protocol (ISRCTN75449581). BMC Musculoskelet Disord, 18(1), 172-172. doi: 10.1186/s12891-017-1513-5.

Gibson, J.N., Waddell, G. (2007). Surgical interventions for lumbar disc prolapse: updated Cochrane Review. Spine 32, 1735-1747. doi: 10.1097/BRS.0b013e3180bc2431.

Green, D. J., Jordan, K. P., Protheroe, J., \& van der Windt, D. (2015). Development of hand phenotypes and changes in hand pain and problems over time in older people. Pain. doi: 10.1097/j.pain.0000000000000402.

Grøvle, L., Haugen, A. J., Hasvik, E., Natvig, B., Brox, J. I., \& Grotle, M. (2014). Patients' ratings of global perceived change during 2 years were strongly influenced by the current health status. Journal of Clinical Epidemiology, 67(5), 508-515. doi:10.1016/j.jclinepi.2013.12.001.

Grovle, L., Haugen, A. J., Keller, A., Natvig, B., Brox, J. I., \& Grotle, M. (2008). Reliability, validity, and responsiveness of the Norwegian versions of the Maine-Seattle Back Questionnaire and the Sciatica Bothersomeness and Frequency Indices. Spine (Phila Pa 1976), 33(21), 2347-2353. doi: 10.1097/BRS.0b013e31818047d6.

Grovle, L., Haugen, A. J., Natvig, B., Brox, J. I., \& Grotle, M. (2013). The prognosis of self-reported paresthesia and weakness in disc-related sciatica. Eur Spine J, 22(11), 2488-2495. doi: 10.1007/s00586-013-2871-9.

Haugen, A. J., Brox, J. I., Grovle, L., Keller, A., Natvig, B., Soldal, D., \& Grotle, M. (2012). Prognostic factors for non-success in patients with sciatica and disc herniation. BMC Musculoskelet Disord, 13.

Haugen, A. J., Grøvle, L., Brox, J. I., Natvig, B., Keller, A., Soldal, D., \& Grotle, M. (2011). Estimates of success in patients with sciatica due to lumbar disc herniation depend upon outcome measure. European Spine Journal, 20(10), 1669-1675. doi: 10.1007/s00586-011-1809-3.

Haugen, A. J., Grovle, L., Keller, A., \& Grotle, M. (2008). Cross-cultural adaptation and validation of the Norwegian version of the Tampa scale for kinesiophobia. Spine (Phila Pa 1976), 33(17), E595-601. doi: 10.1097/BRS.0b013e31817c6c4b.

Harrison, S.A., Stynes, S., Dunn, K.M., Foster, N.E., Konstantinou, K. (2017). Neuropathic Pain in Low Back-Related Leg Pain Patients: What Is the Evidence of Prevalence, Characteristics, and Prognosis in Primary Care? A Systematic Review of the Literature. J Pain, 18(11):1295-1312. doi: 10.1016/j.jpain.2017.04.012.

Hoy, D., March, L., Brooks, P., Blyth, F., Woolf, A., Bain, C., . . Buchbinder, R. (2014). The global burden of low back pain: estimates from the Global Burden of Disease 2010 study. Annals of the Rheumatic Diseases, 73(6), 968. doi: 10.1136/annrheumdis-2013-204428.

Ihlebaek, C., Brage, S., Eriksen HR. (2007). Health complaints and sickness absence in Norway, 19962003. Occup Med (Lond), 1, 43-49.

Jacobs, W.C., van Tulder, M., Arts, M., Rubinstein, S.M., van Middelkoop, M., Ostelo, R.,...Peul W.C. (2001). Surgery versus conservative management of sciatica due to a lumbar herniated disc: a systematic review. Eur Spine J., 20, 513-522.

This article is protected by copyright. All rights reserved. 
Koes, B. W., van Tulder, M. W., \& Peul, W. C. (2007). Diagnosis and treatment of sciatica. BMJ (Clinical research ed.), 334(7607), 1313-1317.

Konstantinou, K., Dunn, K. M., Ogollah, R., Lewis, M., van der Windt, D., \& Hay, E. M. (2018). Prognosis of sciatica and back-related leg pain in primary care: the ATLAS cohort. The Spine Journal, 18(6), 1030-1040. doi: https://doi.org/10.1016/j.spinee.2017.10.071.

Lam, W.W., Loke, A.Y. (2017) Factors and concerns of patients that influence the decision for spinal surgery and implications for practice: A review of the literature. Int J Orthop Trauma Nurs, 25, 11-18. doi: 10.1016/j.ijotn.2016.09.001.

Lin, C., Verwoerd, A., Maher, C., Verhagen, A., Pinto, R., Luijsterburg, P., \& Hancock, M. (2014). How is radiating leg pain defined in randomized controlled trials of conservative treatments in primary care? A systematic review. Eur J Pain, 18(4), 455-64. doi: 10.1002/j.15322149.2013.00384.x.

Luijsterburg, P., Verhagen, A., Ostelo, R., van, d. H., Peul, W., Avezaat, C., \& Koes, B. (2008). Physical therapy plus general practitioners' care versus general practitioners' care alone for sciatica: a randomised clinical trial with a 12-month follow-up. Eur Spine J, 17(4), 509-517.

Lurie, J. D., Tosteson, T. D., Tosteson, A. N. A., Zhao, W., Morgan, T. S., Abdu, W. A., . . Weinstein, J. N. (2014). Surgical versus nonoperative treatment for lumbar disc herniation: eight-year results for the spine patient outcomes research trial. Spine (Phila Pa 1976), 39(1), 3-16. doi: 10.1097/BRS.0000000000000088.

Muthen, B. (2004). Latent variable analysis: Growth mixture modelling and related techniques for longitudinal data. In D. Kaplan (Ed.), Handbook of quantitative methodology for the social sciences. Newbury Park, Ca: Sage.

Nasserinejad, K., van Rosmalen, J., de Kort, W., Lesaffre, E. (2017) Comparison of criteria for choosing the number of classes in Bayesian finite mixture models. PloS one, 12(1):e0168838. doi: 10.1371/journal.pone.0168838

Nijs J., Apeldoorn A., Hallegraeff H., Clark J., Smeets R., Malfliet A.,... Ickmans K. (2015). Low back pain: Guidelines for the clinical classification of predominant neuropathic, nociceptive, or central sensitization pain. Pain Physician, vol./is. 18/3(E333-E346), 1533-3159.

Nylund, K. L., Asparouhov, T., \& Muthén, B. O. (2007). Deciding on the number of classes in latent class analysis and growth mixture modeling: A Monte Carlo Simulation study. Structural Equation Modeling: A Multidisciplinary Journal, 14(4), 535-569. doi: 10.1080/10705510701575396.

Ogollah, R. O., Konstantinou, K., Stynes, S., \& Dunn, K. M. (2018). Determining one-year trajectories of low back related leg pain in primary care patients: growth mixture modelling of a prospective cohort study. Arthritis Care Res (Hoboken), 70(12), 1840-48. doi: 10.1002/acr.23556.

Patrick, D. L., Deyo, R. A., Atlas, S. J., Singer, D. E., Chapin, A., \& Keller, R. B. (1995). Assessing healthrelated quality of life in patients with sciatica. Spine (Phila Pa 1976), 20(17), 1899-908.

Peul, W. C., van Houwelingen, H. C., van den Hout, W. B., Brand, R., Eekhof, J. A., Tans, J. T., . . Koes, B. W. (2007). Surgery versus prolonged conservative treatment for sciatica. N Engl J Med, 356(22), 2245-2256. doi: 10.1056/NEJMoa064039.

Pinto Rz, M., Hancock, M., McLachlan, A. J., \& Koes, B. (2012). Drugs for relief of pain in patients with sciatica: systematic review and meta-analysis. BMJ, Feb 13, 344- e497. doi: 10.1136/bmj.e497.

Sandanger, I., Moum, T., Ingebrigtsen, G., Dalgard, O. S., Sorensen, T., \& Bruusgaard, D. (1998). Concordance between symptom screening and diagnostic procedure: the Hopkins Symptom Checklist-25 and the Composite International Diagnostic Interview I. Soc Psychiatry Psychiatr Epidemiol, 33(7), 345-354.

Sollmann, N., Morrandell, C., Albers, L., Behr, M., Preuss, A., Dinkel, A.,...Krieg, S. (2018) Association of decision-making in spinal surgery with speciality and emotional involvement - the Indications in Spinal Surgery (INDIANA) survey. Acta Neurochirurgica 160(3), 425-438.

This article is protected by copyright. All rights reserved. 
Stynes, S., Konstantinou, K., Ogollah, R., Hay, E. M., \& Dunn, K. M. (2018). Novel approach to characterising individuals with low back-related leg pain: cluster identification with latent class analysis and 12-month follow-up. Pain, 159(4), 728-738. doi: 10.1097/j.pain.0000000000001147.

Stynes, S., Konstantinou, K., \& Dunn, K. M. (2016). Classification of patients with low back-related leg pain: a systematic review. BMC Musculoskelet Disord, 17(1), 1. doi: 10.1186/s12891-0161074-z.

Valat, J.P., Genevay, S., Marty, M., Rozenberg, S., \& Koes, B. (2010). Sciatica. Best Pract Res Clin Rheumatol, .Apr;24(2), 241-52. doi: 10.1016/j.berh.2009.11.005. 24(2).

Vardeh, D., Mannion, R. J., \& Woolf, C. J. (2016). Toward a mechanism-based approach to pain diagnosis. J Pain, 17(9 Suppl), T50-69. doi: 10.1016/j.jpain.2016.03.001.

Verwoerd, A. J., Luijsterburg, P. A., Lin, C. W., Jacobs, W. C., Koes, B. W., \& Verhagen, A. P. (2013). Systematic review of prognostic factors predicting outcome in non-surgically treated patients with sciatica. Eur J Pain, 17(8), 1126-1137. doi: 10.1002/j.1532-2149.2013.00301.x.

Vlaeyen, J. W., Kole-Snijders, A. M., Boeren, R. G., \& van Eek, H. (1995). Fear of movement/(re)injury in chronic low back pain and its relation to behavioral performance. Pain, 62(3), 363-372.

Ware, J. E., (2000). SF-36 health survey update. Spine (Phila Pa 1976), 25(24), 3130.

Table 1 Description of variables used for latent class modelling

Table 2 Statistical indices of fit of the latent cluster models of disc-related sciatica patients Table 3 Baseline characteristics of the total sample and 4 clusters with disc-related sciatica Figure 1 Four cluster latent class analysis solution. Item response probabilities of categorical variables (left vertical axis) and baseline mean leg and back pain intensity (right vertical axis) Figure $\mathbf{2}$ Clinical course over $\mathbf{2 4}$ months of back pain (top figure), leg pain (middle figure) and disability (bottom figure) for the 4 clusters

This article is protected by copyright. All rights reserved. 


\section{Self-report/history items}

Leg pain intensity (0-100): was measured using the visual analogue scale (VAS) $0-100$. Patients were asked to rate their average leg pain during the previous week on 100-mm Visual Analogue Scales (VAS) with $0=$ No pain and $100=$ Maximum pain. For the purpose of latent class modelling, values were converted to a $0-10$ scale.

Back pain intensity (0-100): was measured using the visual analogue scale (VAS) 0-100. Patients were asked to rate their average back pain during the previous week on $100-\mathrm{mm}$ Visual Analogue Scales (VAS) with $0=$ No pain and $100=$ Maximum pain. For the purpose of latent class modelling, values were converted to a $0-10$ scale.

Sensory bothersome (yes/no): A positive response was recorded if patient rated $\geq 4$ out of 6 to the question from the Sciatica Bothersomeness Index (SBI) (Patrick et al., 1995) "in the past week, how bothersome were the symptoms of numbness or tingling in leg, foot or groin on a 0-6 scale where 0 is 'not bothersome' and 6 is extremely bothersome'.

Leg pain worse than back pain (yes/no): A positive response was recorded if the patient's leg pain is worse/ or bothers them more than their back pain, calculated from the leg and back pain intensity scores.

\section{Physical examination items}

Positive straight leg raise test (yes/no): A straight leg raise (SLR) test was defined as positive on reproduction of the patient's leg pain at $\leq 60$ degrees during performance of the SLR test.

Reflex deficit (yes/no): A deficit was defined as either a reduced knee or ankle reflex in the symptomatic lower limb(s).

Myotome deficit (yes/no). A deficit was defined as less than normal muscle strength (grade $5 / 5$ on Oxford manual muscle testing scale) in any of the tested myotome muscle groups of the symptomatic lower limb(s): tiptoe walking (S1-S2); heel walking (L4-L5); hip abduction in standing (L5), knee extension in standing (L3-L4); big toe extension (L5).

Sensory deficit (yes/no): A deficit was defined as either a reduced, significantly reduced or absent (anaesthesia) response to light touch of dermatomal distribution areas in the symptomatic lower $\operatorname{limb}(\mathrm{s})$.

This article is protected by copyright. All rights reserved. 
Table 2 Statistical indices of fit of the latent cluster models of disc-related sciatica patients

\begin{tabular}{|l|l|l|l|l|}
\hline $\begin{array}{l}\text { Number } \\
\text { of } \\
\text { clusters }\end{array}$ & BIC & $\begin{array}{l}\text { Bootstrapped } \\
\text { parametric } \\
\text { LRT p value }\end{array}$ & Entropy & $\begin{array}{l}\text { Smallest } \\
\text { sample size } \\
\text { (\%) }\end{array}$ \\
\hline 2 & 8180.195 & $<0.001$ & 0.825 & $165(35 \%)$ \\
\hline 3 & 8063.765 & $<0.001$ & 0.811 & $134(29 \%))$ \\
\hline 4 & 7976.872 & $<0.001$ & $\mathbf{0 . 8 7 5}$ & $\mathbf{5 9 ( 1 2 \% )}$ \\
\hline 5 & 7950.368 & $<0.001$ & 0.875 & $62(13.3 \%)$ \\
\hline 6 & 7927.056 & $<0.001$ & 0.895 & $35(7.5 \%)$ \\
\hline BIC Bayesian Information Criteria; LRT likelihood ratio test. \\
a The number (proportion) of patients in the smallest class; at least \\
$10 \%$ of sample should be in each class. The bold text indicates the \\
model selected as having the optimal number of clusters
\end{tabular}

This article is protected by copyright. All rights reserved. 
Table 3 Baseline characteristics of the total sample and 4 clusters with disc-related sciatica

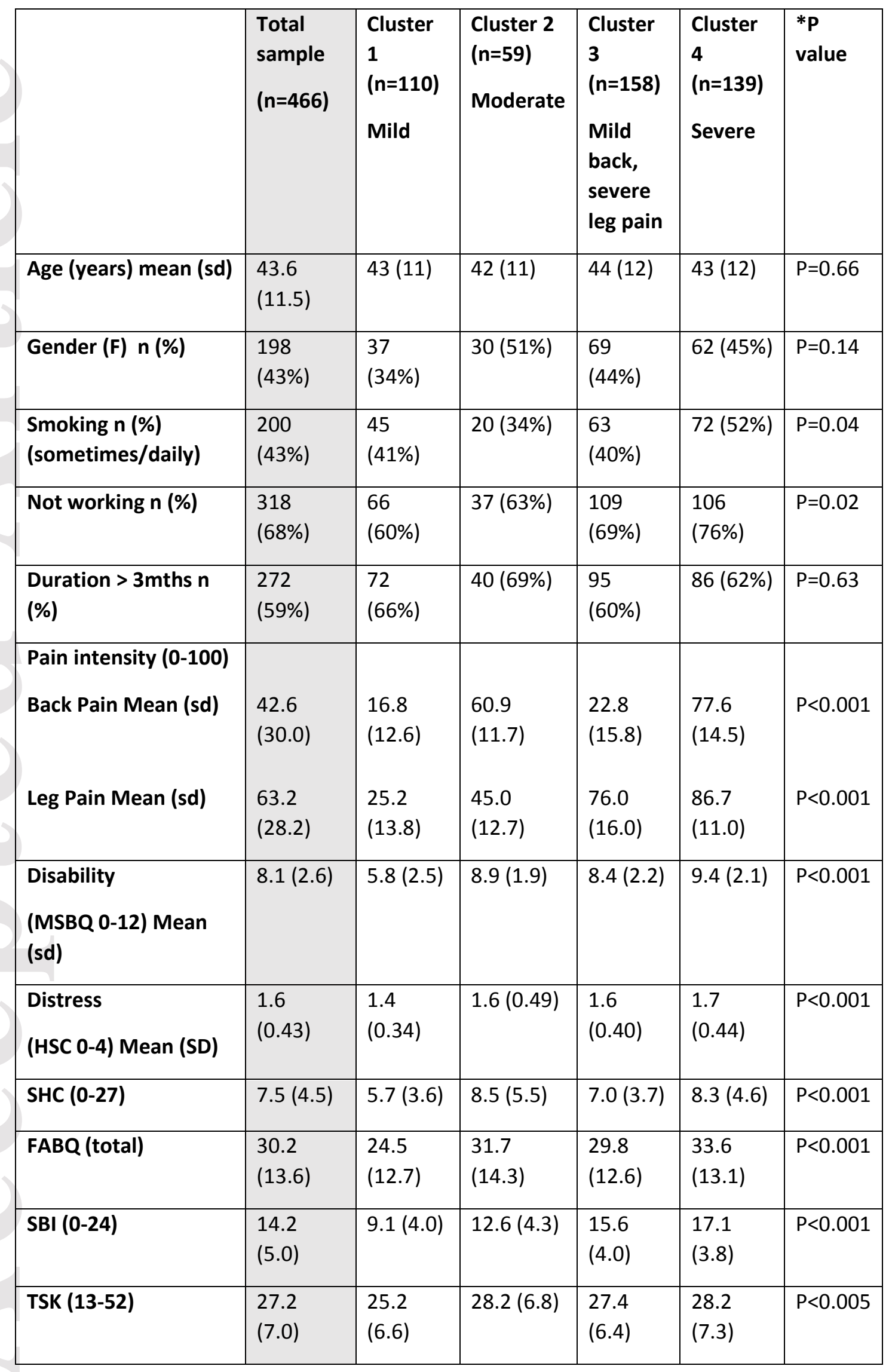

This article is protected by copyright. All rights reserved. 


\begin{tabular}{|c|c|c|c|c|c|c|}
\hline SF36 General health & $\begin{array}{l}69.2 \\
(20.3)\end{array}$ & \begin{tabular}{|l|}
74.0 \\
$(17.6)$
\end{tabular} & $\begin{array}{l}69.0 \\
(20.1)\end{array}$ & \begin{tabular}{|l|}
70.2 \\
$(18.9)$
\end{tabular} & \begin{tabular}{|l|}
66.5 \\
$(22.0)$
\end{tabular} & $P<0.012$ \\
\hline SF36 Bodily Pain & $\begin{array}{l}22.7 \\
(17.7)\end{array}$ & $\begin{array}{l}37.1 \\
(19.5)\end{array}$ & $\begin{array}{l}25.0 \\
(13.4)\end{array}$ & \begin{tabular}{|l|}
19.6 \\
$(14.6)$
\end{tabular} & \begin{tabular}{|l|}
13.9 \\
$(13.1)$
\end{tabular} & $P<0.001$ \\
\hline $\begin{array}{l}\text { SF36 Physical } \\
\text { function }\end{array}$ & $\begin{array}{l}49.7 \\
(25.4)\end{array}$ & \begin{tabular}{|l|}
70.8 \\
$(16.6)$
\end{tabular} & $\begin{array}{l}48.7 \\
(19.2)\end{array}$ & \begin{tabular}{|l|}
46.7 \\
$(24.7)$
\end{tabular} & \begin{tabular}{|l|}
40.9 \\
$(24.5)$
\end{tabular} & $P<0.001$ \\
\hline $\begin{array}{l}\text { Level of disc } \\
\text { herniation (imaging } \\
\text { confirmed) } \\
\text { L2/3 } \\
\text { L3/4 } \\
\text { L4/5 } \\
\text { L5/S1 } \\
\text { Other }\end{array}$ & $\begin{array}{l}4(1 \%) \\
10(2 \%) \\
172(37 \%) \\
233(51 \%) \\
41(9 \%)\end{array}$ & $\begin{array}{l}0(0 \%) \\
2(2 \%) \\
39(36 \%) \\
58(53 \%) \\
10(9 \%)\end{array}$ & $\begin{array}{l}2(3 \%) \\
1(2 \%) \\
20(34 \%) \\
30(51 \%) \\
6(10 \%)\end{array}$ & $\begin{array}{l}1(1 \%) \\
1(1 \%) \\
59(39 \%) \\
81(52 \%) \\
11(7 \%)\end{array}$ & $\begin{array}{l}1(1 \%) \\
6(4 \%) \\
54(39 \%) \\
64(46 \%) \\
14(10 \%)\end{array}$ & $P=0.6$ \\
\hline \multicolumn{7}{|c|}{$\begin{array}{l}\text { *P value, Probability value tested across clusters } 1 \text { to } 4 \text { (not including total sample); sd, } \\
\text { standard deviation; F, female; MSBQ Maine Seattle Back Questionnaire; HSC Hopkins } \\
\text { Symptom Check List; SHC, Subjective Health Complaints; FABQ, Fear Avoidance Beliefs } \\
\text { Questionnaire; SBI, Sciatica Bothersomeness Index; TSK, Tampa Scale for Kinesiophobia; }{ }^{{ }^{4}} \\
\text { herniation at more than one disc level, lumbotransitional vertebra, other causes. }\end{array}$} \\
\hline
\end{tabular}

This article is protected by copyright. All rights reserved. 
Figure 1 Four cluster latent class analysis solution. Item response probabilities of categorical variables (left vertical axis) and baseline mean leg and back pain intensity (right vertical axis)

Figure $\mathbf{2}$ Clinical course over $\mathbf{2 4}$ months of back pain (top figure), leg pain (middle figure) and disability (bottom figure) for the 4 clusters

Figure 1 Four cluster latent class analysis solution. Item response probabilities of categorical variables (left vertical axis) and baseline mean leg and back pain intensity (right vertical axis)

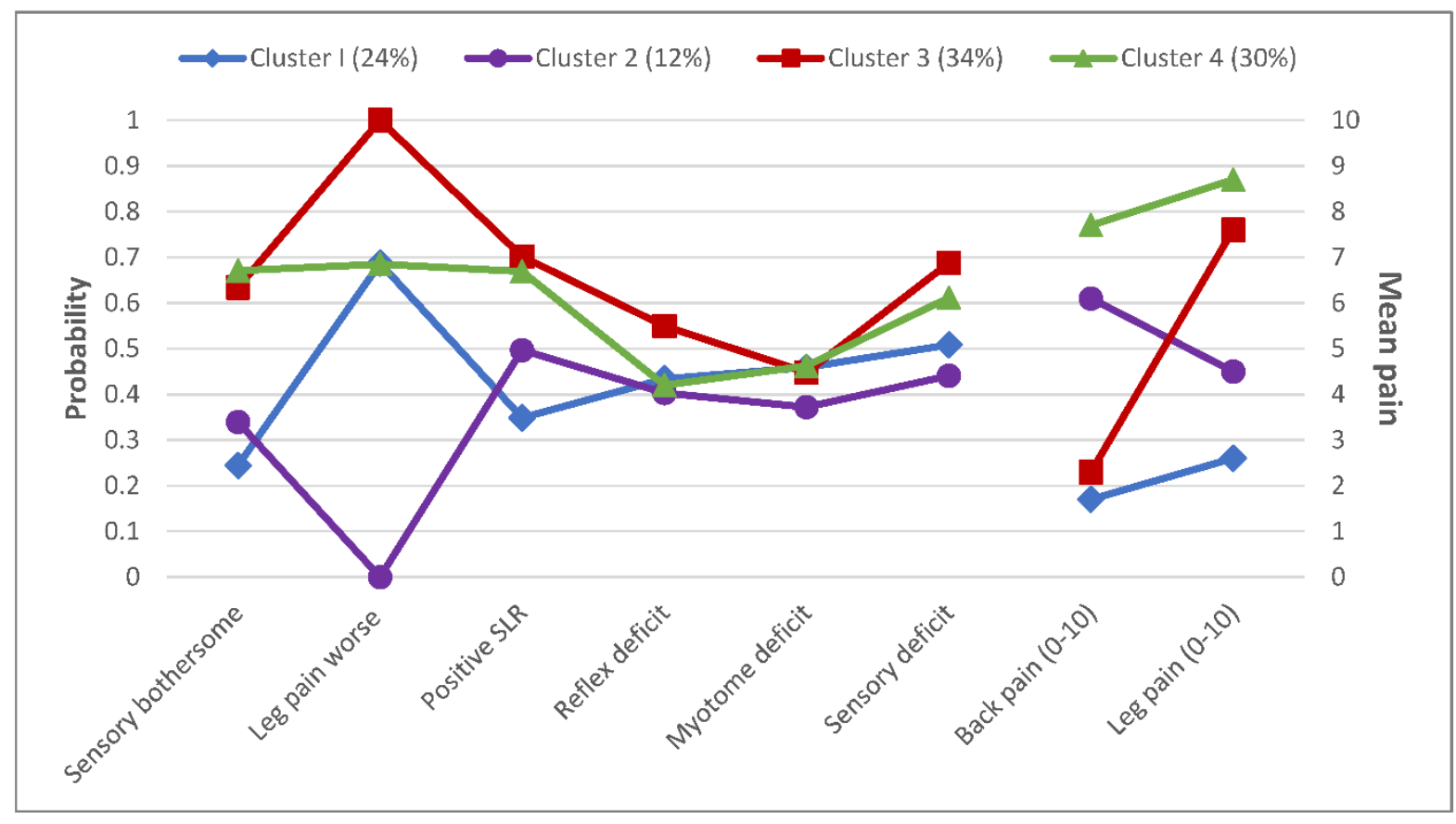

This article is protected by copyright. All rights reserved. 
Figure $\mathbf{2}$ Clinical course over $\mathbf{2 4}$ months of back pain (top figure), leg pain (middle figure) and disability (bottom figure) for the 4 clusters
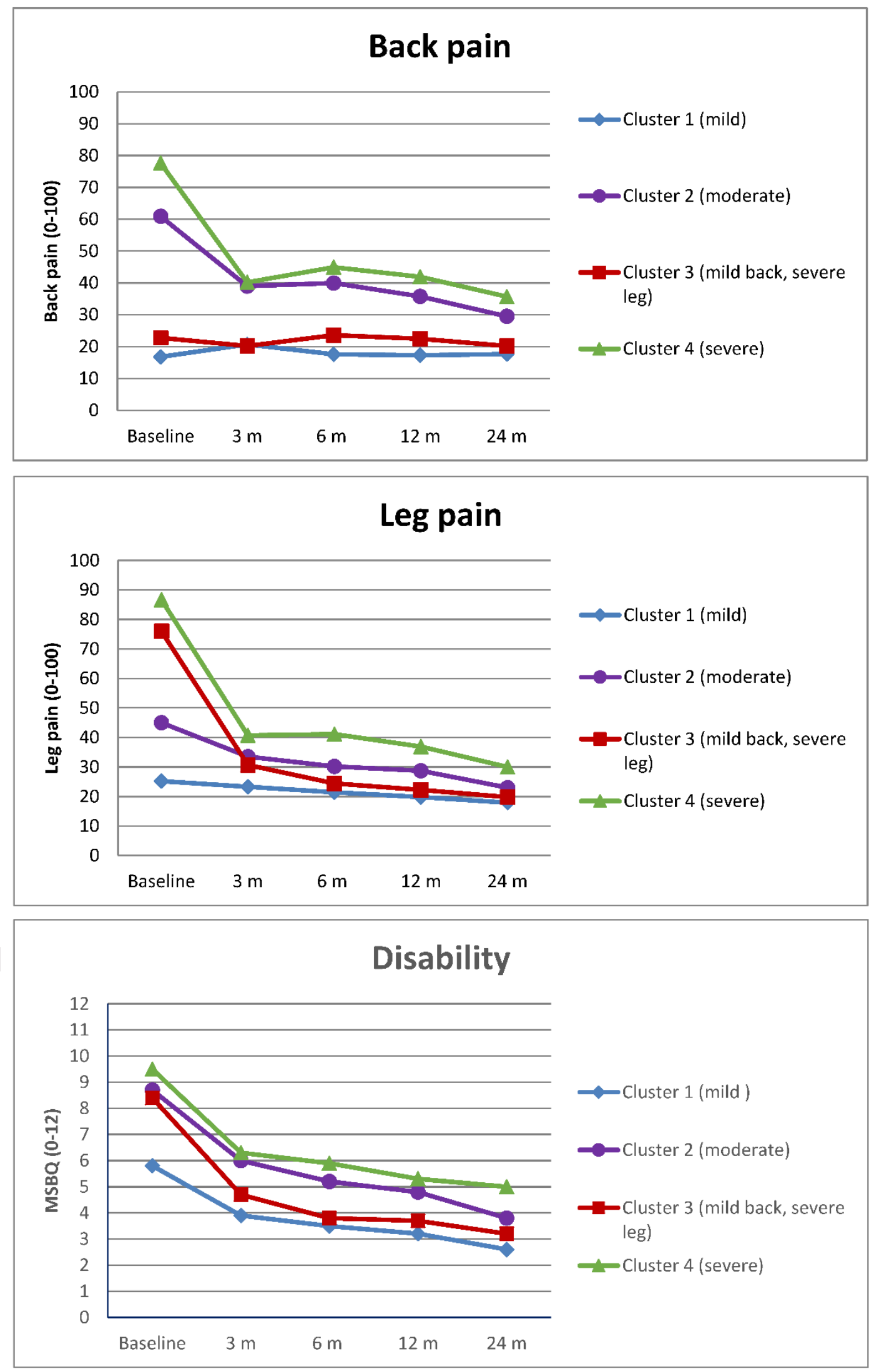

This article is protected by copyright. All rights reserved. 